\title{
Dynamic Response of a Period Viaduct In-plane Vibration Under Spatially Non-uniform Seismic Wave
}

\author{
Huang Qian, Sheng Guo-jun, XU Man-qing, Zhao Jiang-qian \\ Nanchang Institute of Technology, Nanchang, Jianxi, 330029, China
}

\section{KEYWORD: Floquet transform method; viaduct; period structure; energy bands; non-uniform seismic wave}

ABSTRACT: By using the Floquet transform method and based on the periodic structure's theory, a theoretical model has been developed to determine the dynamic response of the periodic viaduct accounted for the non-uniform seismic excitations at different piers of the viaduct in this study. Numerical results show that there are three kinds of characteristic waves propagating in the periodic viaduct for the in-plane vibrations of the viaduct. The first kind of wave is a highly decaying wave; the second kind of wave can only propagate at a very narrow frequency range; the third kind of wave may propagate in pass bands with small attenuation. Moreover, when the periodic viaduct is subjected to passband seismic waves, the disturbance will propagate over a very long distance and the mode as well as attenuation of the wave motion far from the source is determined by the characteristic wave with the smallest attenuation. When the periodic viaduct is subjected to stopband seismic waves, the disturbance will restricted to the forced vibration region.

\section{INTRODUCTION}

Viaduct structure is effective to solve the displacement and settlement of soft ground in the highspeed rail, and widely used in engineering. In order to facilitate the design and construction, viaduct structure generally have equal span, therefore, when the distance between adjacent piers of multispan viaduct structure is constant, it can be considered as periodic structure, the basic element consists of three parts: a pier, two longitudinal beams and beam-beam spring, beam-pier springs.

Research [1,2] shows that periodic structure has a significant vibration characteristics, that is, energy band exists in periodic structure, when elastic wave propagate in periodic structures, vibration within certain frequency range cannot be passed, called band gap; and vibration within certain frequency range can be passed, called pass band. It provides a new idea for the seismic design and vibration control by using vibration characteristics of periodic structures. For example, designing rational geometry and material parameters of the periodic viaduct, it ensure the main seismic waves are in pass band of the viaduct structure itself, otherwise, greater energy seismic waves pass difficultly the viaduct structure, causing sharp energy increase and structural damage. So considering the energy band principle of periodic structures, the seismic design and vibration control measures can be achieved by adjusting the structure itself, no other additional structures.

At present, there are more seismic design methods about viaduct, such as: the response spectrum method [4, 5], the earthquake time-history analysis [6, 7] and random vibration method [8, 9]. Above methods are generally to simplify viaduct as single degree of freedom system or multi-degree of freedom system, seismic wave simulated by standing wave, the corresponding vibration control equations obtained and solved. Clearly, the analysis of standing wave cannot reflect the propagation characteristics of vibration wave in the periodic viaduct, and when seismic wave causing structural vibration, the first excited vibration is viaduct piers in soft foundation, the seismic energy input to the viaduct structure varies with the distance from the viaduct pier and vibration source location, showed nonlinear spatial distribution $[10,11]$.

Therefore, it is necessary to establish mathematical model which can reflect propagation characteristics of non-uniform seismic wave in the periodic viaduct, providing theoretical basis for seismic design of the viaduct structure. 
In the paper, in order to deal with the non-uniform seismic excitation of the piers in multi-span viaduct structure, the Floquet transform method is introduced to decompose the non-uniform seismic excitation into a set of spatially harmonic waves. The periodic viaduct is assumed to be composed of an infinite number of spans, and each span is supposed to consist of a pier, two longitudinal beams and beam-beam spring, beam-pier springs. For the in-plane vibration of the viaduct, the eigen equation of the periodic viaduct in the wave-number domain is obtained by invoking the periodic condition of the viaduct and using transfer matrix method. By means of the inverse Floquet transform, the space-domain vibration characteristics of the periodic viaduct to non-uniform seismic waves can be retrieved, and the influence caused by the characteristic wave propagating in periodic structures and the different seismic waves discussed.

\section{FLOQUET TRANSFORM METHOD AND PERIODIC VIADUCT MODEL}

The displacement amplitude in the bottom of the piers vary with the different spatial locations of periodic structure piers in the action of seismic waves, and the displacive phase transition between adjacent piers is uncertain, thus, the periodic viaduct structural dynamic response is caused by a series of non-uniform waves, which cannot be directly used to analysis periodic viaduct. Therefore, the Floquet transform method is introduced to convert the non-uniform waves to spatially harmonic waves. For periodic viaduct in-plane vibration, $L$ is assumed a lattice vector cycle of adjacent lattice points in the one-dimensional direction, according to literature [13], lattice vector $\mathrm{R}$ can be expressed by $\mathbf{R}=n L e$, where, e is basis vector of the one-dimensional direction. If $f(\mathbf{R})=f(n L)$ is discrete spatial domain functions of one-dimensional vector, the Floquet transform and inverse transform are defined as follows [14],

$$
\left.\begin{array}{l}
F(f(n L))=\hat{f}(\kappa)=\sum_{n=-\infty}^{+\infty} f(n L) e^{\mathrm{i} \kappa n L} \\
f(n L)=\frac{L}{2 \pi} \int_{-\pi / L}^{\pi / L} \hat{f}(\kappa) e^{-\mathrm{i} \kappa n L} d \kappa
\end{array}\right\}
$$

Where, $\kappa$ is the number of lattice waves, the superscript $" \wedge "$ means wave-number domain.

According to the formula (1), the Floquet transform of discrete spatial sequence function $f[(m+n) L$ can be expressed as,

$$
F(f[(m+n) L])=\hat{f}^{(n)}(\kappa)=\sum_{m=-\infty}^{+\infty} f[(m+n) L] e^{\mathrm{i} \kappa m L}=e^{-\mathrm{i} \kappa n L} \hat{f}(\kappa)
$$

Considering the viaduct wave field solution under the action of seismic wave, the Fourier transform between time $t$ and frequency domain $\omega$ are defined as follows:

$$
\left.\begin{array}{l}
\bar{f}(\omega)=\int_{-\infty}^{+\infty} f(t) e^{-i \omega t} d t \\
f(t)=\frac{1}{2 \pi} \int_{-\infty}^{+\infty} \bar{f}(\omega) e^{\mathrm{i} \omega t} d \omega
\end{array}\right\}
$$

where, the superscript "-" indicates the frequency domain.

Using equations (2) and (3), the viaduct structural dynamic response under seismic waves can be transformed into harmonic wave dynamic response of frequency domain - wave number domain, its amplitude is $\hat{f}(\kappa)$.

Fig. 1 shows periodic viaduct structure with infinite number of spans, track, track plates and beam of each span are simplified for the left or right horizontal beam, connected by a pier, piers supported on a rigid semi-infinite saturated soil body, track connecting the left and right horizontal beam simulated by spring, the jointing element between piers and the left or right horizontal beam simulated also by springs, therefore, each unit of periodic viaduct includes a pier, two horizontal beams and three springs. 
Considering periodic viaduct in-plane vibration under the seismic wave, according to EulerBernoulli beam theory [13], for the pier of n-span element of viaduct, its motion equations in frequency domain - wave number domain are as follows,

$$
\left.\begin{array}{l}
E_{d} \partial^{2} \hat{\bar{u}}_{d}^{(n)}(\kappa, z) / \partial z^{2}+\rho_{d} \omega^{2} \hat{\bar{u}}_{d}^{(n)}(\kappa, z)=0 \\
E_{d} I_{d} \partial^{4} \hat{\bar{v}}_{d}^{(n)}(\kappa, z) / \partial z^{4}-\rho_{d} A_{d} \omega^{2} \hat{\bar{v}}_{d}^{(n)}(\kappa, z)=0
\end{array}\right\}
$$

where, $E_{d}, \rho_{d}, A_{d}$ and $I_{d}$ are respectively elastic modulus, density, cross-sectional area and rotational inertia of piers, $\hat{\bar{u}}_{d}^{(n)}$ and $\hat{\bar{v}}_{d}^{(n)}$ are respectively axial and tangential displacement of piers in frequency domain - wave number domain.

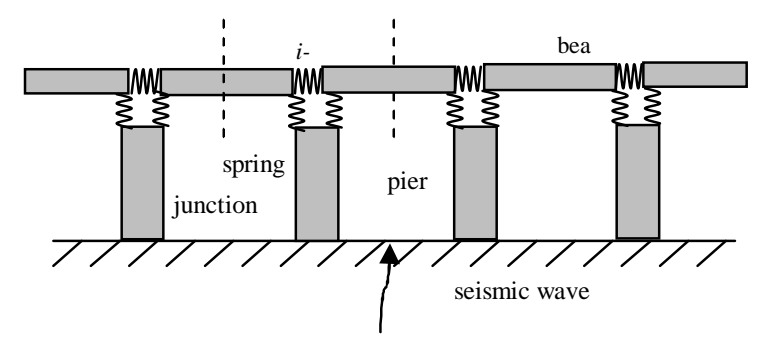

Fig.1 A schematic illustration of a periodic viaduct with spring junction subjected to seismic wave

For the pier of $\mathrm{n}$-span, the state vector $\hat{\overline{\mathbf{\Psi}}}_{d}^{(n)}(\kappa, z)$ of arbitrary position $\mathrm{Z}$ is composed of displacement vector $\hat{\mathbf{q}}_{d}^{(n)}(\kappa, z)$ and internal force vector $\hat{\overline{\mathbf{f}}}_{d}^{(n)}(\kappa, z)$, its expression is,

$$
\left.\begin{array}{l}
\hat{\overline{\mathbf{\psi}}}_{d}^{(n)}(\kappa, z)=\left\{\hat{\overline{\mathbf{q}}}_{d}^{(n)}(\kappa, z), \hat{\overline{\mathbf{f}}}_{d}^{(n)}(\kappa, z)\right\}^{T} \\
\hat{\overline{\mathbf{q}}}_{d}^{(n)}(\kappa, z)=\left\{\hat{\bar{u}}_{d}^{(n)}(\kappa, z), \hat{\bar{v}}_{d}^{(n)}(\kappa, z), \hat{\bar{\theta}}_{d}^{(n)}(\kappa, z)\right\}^{T} \\
\hat{\mathbf{f}}_{d}^{(n)}(\kappa, z)=\left\{\hat{\bar{N}}_{d}^{(n)}(\kappa, z), \hat{\bar{Q}}_{d}^{(n)}(\kappa, z), \hat{\bar{M}}_{d}^{(n)}(\kappa, z)\right\}^{T}
\end{array}\right\}
$$

When the periodic viaduct belongs to in-plane vibration under the seismic wave, viaduct horizontal beams are also in-plane vibration, Euler-Bernoulli beam theory [13] can be used, the motion equations of horizontal beam in frequency domain - wave number domain are as follows,

$$
\left.\begin{array}{l}
E_{b} \partial^{2} \hat{\bar{u}}_{b}^{(n)}(\kappa, x) / \partial x^{2}+\rho_{b} \omega^{2} \hat{\bar{u}}_{b}^{(n)}(\kappa, x)=0 \\
E_{b} I_{b} \partial^{4} \hat{\bar{v}}_{b}^{(n)}(\kappa, x) / \partial x^{4}-\rho_{b} A_{b} \omega^{2} \hat{\bar{v}}_{b}^{(n)}(\kappa, x)=0
\end{array}\right\}
$$

where, $E_{b}, \rho_{b}, A_{b}$ and $I_{b}$ are respectively elastic modulus, density, cross-sectional area and rotational inertia of horizontal beams, $\hat{\bar{u}}_{b}^{(n)}$ and $\hat{\bar{v}}_{b}^{(n)}$ are respectively axial and tangential displacement of horizontal beams in frequency domain - wave number domain.

According to Euler-Bernoulli beam theory [13], Transfer matrix of pier and horizontal beam can be deduced.

For beam-beam junction of n-span, assuming $k_{t}^{(t)}, k_{t}^{(s)}$ and $k_{t}^{(b)}$ are respectively axial compression stiffness, shear stiffness and rotational stiffness of beams-beam junction spring, then the axial force $\hat{\bar{N}}_{t}^{(n)}(\kappa)$, shear force $\hat{\bar{Q}}_{t}^{(n)}(\kappa)$ and bending moment $\hat{\bar{M}}_{t}^{(n)}(\kappa)$ of beams-beam junction spring in frequency domain - wave number domain are expressed as,

$$
\left.\begin{array}{l}
\hat{\bar{N}}_{t}^{(n)}(\kappa)=k_{t}^{(t)}\left[\hat{\bar{u}}_{b}^{(n)}\left(\kappa, 0^{+}\right)-\hat{\bar{u}}_{b}^{(n)}\left(\kappa, 0^{-}\right)\right] \\
\hat{\bar{Q}}_{t}^{(n)}(\kappa)=k_{t}^{(s)}\left[\hat{\bar{v}}_{b}^{(n)}\left(\kappa, 0^{+}\right)-\hat{\bar{v}}_{b}^{(n)}\left(\kappa, 0^{-}\right)\right] \\
\hat{\bar{M}}_{t}^{(n)}(\kappa)=-k_{t}^{(b)}\left[\hat{\bar{\theta}}_{b}^{(n)}\left(\kappa, 0^{+}\right)-\hat{\bar{\theta}}_{b}^{(n)}\left(\kappa, 0^{-}\right)\right]
\end{array}\right\}
$$

where, $0^{-}$and $0^{+}$are respectively the left and the right of the junction spring. 


\section{DYNAMIC RESPONSE OF A PERIODIC VIADUCT IN-PLANE VIBRATION}

Using Equation (2), the displacement vector $\hat{\overline{\mathbf{q}}}_{d}^{(n)}\left(\kappa, L_{d}\right)$ at the bottom of viaduct pier can be deduced under the seismic waves, through the transfer matrix of pier, the displacement vector $\hat{\mathbf{q}}_{d}^{(n)}\left(\kappa, 0^{+}\right)$and the internal force vector at the top of pier can be deduced,

$$
\hat{\mathbf{q}}_{d}^{(n)}\left(\kappa, 0^{+}\right)=\mathbf{C}_{d} \hat{\mathbf{f}}_{d}^{(n)}\left(\kappa, 0^{+}\right)+\mathbf{T}_{q q}^{(d)-1}\left(L_{d}\right) \hat{\overline{\mathbf{q}}}_{d}^{(n)}\left(\kappa, L_{d}\right)
$$

where, $\quad \mathbf{C}_{d}=-\mathbf{T}_{q q}^{(d)-1}\left(L_{d}\right) \mathbf{T}_{q f}^{(d)}\left(L_{d}\right), \quad \mathbf{T}^{(d)}\left(L_{d}\right)=\left[\begin{array}{c}\mathbf{T}_{q q}^{(d)}\left(L_{d}\right) \mathbf{T}_{q f}^{(d)}\left(L_{d}\right) \\ \mathbf{T}_{f q}^{(d)}\left(L_{d}\right) \mathbf{T}_{f f}^{(d)}\left(L_{d}\right)\end{array}\right], \quad T_{q q}^{(d)}\left(L_{d}\right)$ and $\mathbf{T}_{q f}^{(d)}\left(L_{d}\right)$ are respectively sub-matrix of the pier transfer matrix .

Formula (7) is expressed in matrix form,

$$
\text { where, } \mathbf{E}_{l}^{(a)}=\left[\begin{array}{ccc}
0 & 1 & 0 \\
-1 & 0 & 0 \\
0 & 0 & -1
\end{array}\right], \mathbf{E}_{r}^{(a)}=\left[\begin{array}{ccc}
0 & -1 & 0 \\
1 & 0 & 0 \\
0 & 0 & 1
\end{array}\right] . \quad \hat{\overline{\mathbf{f}}}_{d}^{(n)}\left(\kappa, 0^{+}\right)=\mathbf{E}_{l}^{(a)} \hat{\mathbf{f}}_{b}^{(n)}\left(\kappa, 0^{-}\right)+\mathbf{E}_{r}^{(a)} \hat{\overline{\mathbf{f}}}_{b}^{(n)}\left(\kappa, 0^{+}\right)
$$

In terms of equations (6) (9), the internal force vectors of the left and the right beam in $n$-span unit are deduced,

$$
\left.\begin{array}{r}
\hat{\overline{\mathbf{f}}}_{b}^{(n)}\left(\kappa, 0^{-}\right)=\mathbf{J}_{l l} \hat{\overline{\mathbf{q}}}_{b}^{(n)}\left(\kappa, 0^{-}\right)+\mathbf{J}_{l r} \hat{\mathbf{q}}_{b}^{(n)}\left(\kappa, 0^{+}\right)+\mathbf{J}_{l d} \hat{\mathbf{q}}_{d}^{(n)}\left(\kappa, 0^{+}\right) \\
\hat{\mathbf{f}}_{b}^{(n)}\left(\kappa, 0^{+}\right)=\mathbf{J}_{r l} \hat{\overline{\mathbf{q}}}_{b}^{(n)}\left(\kappa, 0^{-}\right)+\mathbf{J}_{r r} \hat{\mathbf{q}}_{b}^{(n)}\left(\kappa, 0^{+}\right)+\mathbf{J}_{r l} \hat{\mathbf{q}}_{d}^{(n)}\left(\kappa, 0^{+}\right)
\end{array}\right\}
$$

The characteristic equation of periodic viaduct in-plane vibration can be obtained,

$$
\left[\mathbf{T}^{(b)}\left(L_{b} / 2\right) \mathbf{S}_{J} \mathbf{T}^{(b)}\left(L_{b} / 2\right)-e^{-\mathrm{i \kappa} \kappa_{\alpha} L} \mathbf{I}_{6 \times 6}\right] \hat{\bar{\Psi}}_{b}^{(n)}\left(\kappa,-L_{b} / 2\right)=0
$$

where, $\kappa_{\alpha}$ is complex wave number of wave propagation in periodic viaduct, the real part means the phase transition of lattice wave, and the imaginary part indicates wave decay in periodic viaduct

Using formula (11), the state vector of the left beam can be got in the frequency domain - wave number domain,

then by the formula (1), $\overline{\boldsymbol{\psi}}_{b}^{(n)}\left(n L_{b},-L_{b} / 2\right)$ in the frequency domain - spatial domain can be obtained by means of the inverse Floquet transform.

$$
\overline{\boldsymbol{\psi}}_{b}^{(n)}\left(n L_{b},-L_{b} / 2\right)=\frac{L_{b}}{2 \pi} / \int_{-\pi / L_{b}}^{\pi / L_{b}} \hat{\overline{\boldsymbol{\psi}}}_{b}^{(n)}\left(\kappa,-L_{b} / 2\right) d \kappa=\frac{L_{b}}{2 \pi} / \int_{-\pi / L_{b}}^{\pi / L_{b}} \hat{\overline{\boldsymbol{\psi}}}_{b}\left(\kappa,-L_{b} / 2\right) e^{-\mathrm{i} n \kappa L_{b}} d \kappa
$$

\section{NUMERICAL RESULTS}

In order to research the dynamic response of periodic viaduct under non-uniform seismic wave, as shown in Fig.1. The vibration source adopts unit circular harmonic loads in saturated soil to simulate seismic wave field. The coordinate of unit circle center $\left(x_{s}, y_{s}, z_{s}\right)$ is $(0.0,20.0 \mathrm{~m}, 10.0 \mathrm{~m})$, radius $\mathrm{R}$ is 0.5 , load amplitude $\mathrm{Fz}$ is $1.0 \mathrm{~N}$. Saturated soil parameters are as follows, $\mu=2.0 \times 10^{7} \mathrm{~N} / \mathrm{m}^{2}$,

$$
\begin{aligned}
& a_{\infty}=2.0, \quad \lambda=4.0 \times 10^{7} \mathrm{~N} / \mathrm{m}^{2}, \quad \alpha=0.97, \quad b_{p}=1.94 \times 10^{6} \mathrm{~kg} / \mathrm{m}^{3} \cdot \mathrm{s}, \quad M=2.4 \times 10^{8} \mathrm{~N} / \mathrm{m}^{2}, \quad \phi=0.4, \\
& \rho_{s}=2.0 \times 10^{3} \mathrm{~kg} / \mathrm{m}^{3}, \quad \rho_{f}=1.0 \times 10^{3} \mathrm{~kg} / \mathrm{m}^{3} .
\end{aligned}
$$


Using equation (12), characteristic wave of periodic viaduct in-plane vibration can be obtained, shown in Fig. 2.

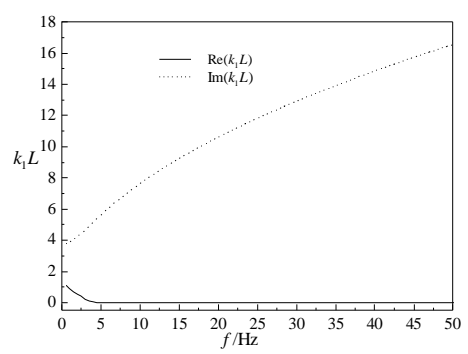

(a)

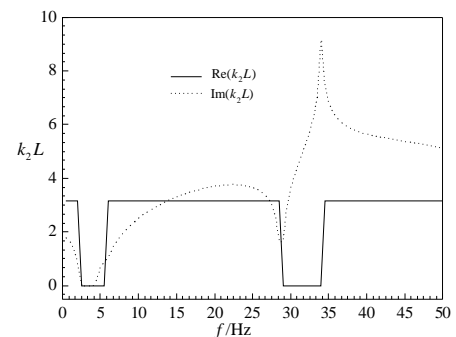

(b)

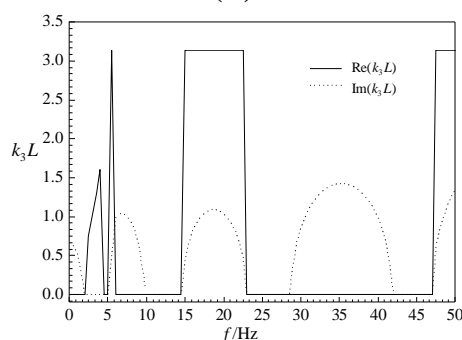

(c)

Fig. 2 The energy bands for the characteristic waves of the periodic viaduct undergoing in-plane motion

As is shown in Fig.2, there are three kinds of characteristic wave when the periodic viaduct undergoes in-plane motion, the distribution of their energy bands varies with different frequencies. The imaginary part of the first and the second kind of waves is larger, which indicates the first and second kind of waves cannot propagate far in periodic viaduct, and vibration waves decay rapidly.

The imaginary part of the third kind of waves shows alternating pass band and band gap at different frequencies, and be smaller than that of above two kinds, which indicates the third kind of waves is mainly propagated in viaduct vibration.

\section{CONCLUSION}

In the thesis, combining the Floquet transform and the transfer matrix method, using the periodicity of viaduct structure, the dynamic response of periodic viaduct in-plane vibration under spatial non-uniform seismic wave is analyzed. In the frequency domain - wave number domain, the periodical structure model of beam-beam and beam-pier connected by springs is established, the transfer matrixes of spring junctions, horizontal beams and pier are deduced, the characteristic equation of periodical viaduct formed and the vibration control equations of viaduct structure established, finally, the solution of spatial domain is got by the inverse Floquet transform. Using numerical examples, the propagation characteristics of waves and the vibration characteristics of periodical viaduct in spatial non-uniform seismic wave are discussed, the numerical results show that, There are three kinds of wave existed in periodic viaduct, the first kind of wave decays rapidly, the second kind of wave 
propagates only in a limited frequency domain, the third kind of wave can propagate in the pass band and decay slowly.

\section{ACKNOWLEDGMENTS}

The project is supported by the National Natural Science Foundation of China with grant number No. 51269021, 51569016. Also, the research is supported by the Jiangxi province science and technology plan projects(No: 20133ACB20006, 20141BBG70088). Moreover, the financial support from Science and technology project of Jiangxi Provincial Education Department Number GJJ14755 is greatly appreciated.

\section{REFERENCE}

[1] Sigalas MM, Economou EN Elastic and acoustic waveband structure[J]. Journal of Sound and Vibration, 1992, 158:377-382.

[2] Kushwaha MS, Halevi P, Dobrzynski L, et al. Acoustic band structure of periodic elastic composites[J]. Physical Review Letters, 1993, 71:2022-2025.

[3] Xu B, Lu JF, Wang JH Dynamic response of an infinite beam overlying a layered poroelastic half space to moving loads[J]. Journal of Sound and Vibration, 2007, 306: 91-110.

[4] Kiureghian AD, Neuenhofer A Response spectrum method for multi-support seismic excitations[J]. Earthquake Engineering \& Structural Dynamics. 1992, 21: 713-740.

[5] Singh M, Maldonado G An improved response spectrum method for calculating seismic design response: classically damped structures[J]. Earthquake Engineering \& Structural Dynamics. 1991, 20: 621-635.

[6] Nazmy AS, Abdel-Ghaffar AM Non-linear earthquake-response analysis of long-span cablestayed bridges: theory[J]. Earthquake Engineering \& Structural Dynamics. 1990, 19:45-62.

[7] Ingham TJ, Rodriguez S, Nader M Nonlinear analysis of the Vincent Thomas Bridge for seismic retrofit[J]. Computers \& Structures 1997, 64: 1221-1238.

[8] Lin JH, Zhang YH Seismic spatial effects for long-span bridges using the pseudo excitation method[J]. Engineering Structures 2004, 26: 1207-1216.

[9] Soyluk K Comparison of random vibration methods for multi-support seismic excitation analysis of long-span bridges[J]. Engineering Structures. 2004, 26:1573-1583.

[10]Biot MA Theory of propagation of elastic waves in a fluid-saturated porous solid. II: Higher frequency range[J]. Journal of the Acoustical Society of America, 1956, 28(2): 179-191.

[11]Biot MA Mechanics of deformation and acoustic propagation in porous media[J]. Journal of Applied Physics, 1962, 33(4): 1482-1498.

[12] Lu JF, Jeng DS, Nie WD Dynamic response of a pile embedded in a porous medium and subjected to plane SH waves[J]. Computers and Geotechnics, 2006, 33: 404-418.

[13]Wen Jihong, Yu Dianlong, Wang Gang, etc. Elastic wave band gaps in flexural vibrations of straight beams[J]. Chinese journal of Mechanical Engineering, 2005, 41: (4) 1-6.

[14]Kittel C Introduction to Solid State Physics[M]. Wiley, New York, 1996.

[15]Jin B, Zhong Z. Lateral dynamic compliance of pile embedded in poroelastic half space[J]. Soil Dynamic and Earthquake Engineering, 2001, 21: 519-525.

[16] Yang guitong, Zhang shanyuan. Elastodynamics [M].Beijing, 1988. 
[17] Mead DJ, Yaman Y The response of infinite periodic beams to point harmonic forces: a flexural wave analysis[J]. Journal of Sound and Vibration, 1991, 144: 507-530.

[18] Mead DJ, Pujara KK Space-harmonic analysis of periodically supported beams: response to convicted random loading[J]. Journal of Sound and Vibration, 1971, 14: 525-532. 